\title{
How Causal Structural Knowledge Adds Decision-Support in Monitoring of Automotive Body Shop Assembly Lines
}

\author{
Johannes Huegle, Christopher Hagedorn and Matthias Uflacker \\ Hasso Plattner Institute, University of Potsdam, Potsdam, Germany \\ johannes.huegle@hpi.de,christopher.hagedorn@hpi.de, matthias.uflacker@hpi.de
}

\begin{abstract}
The efficiency of modern automotive body shop assembly lines is highly related to the reduction of downtimes due to failures and quality deviations within the manufacturing process. Consequently, the need for implementing tools into the assembly lines for on-line monitoring, and failure diagnosis, also under the prism of improving the troubleshooting, is of great importance. While the identification of root causes and elimination of failures is usually built upon individual on-site expert knowledge, causal graphical models (CGMs) have opened the possibility to make a purely data-driven assessment. In this demo, we showcase how a CGM of the production process is incorporated into a monitoring tool to function as a decision-support system for an operator of a modern automotive body shop assembly line and enables fast and effective handling of failures and quality deviations.
\end{abstract}

\section{Background}

Modern automotive manufacturing enterprises have to cope with growing demands for increased product quality, greater product variability, shorter product life-cycles, reduced cost, and global competition [Liang et al., 2004]. In order to meet these demands, modern car body shop assembly lines are highly optimized and operative with a minimum of human interface. Hence, the occurrence of failures and deviations of quality measurements that require a human intervention are a major cause of unscheduled stoppage of the car body assembly line, and are costly not only in terms of time lost, but also in terms of capital destroyed [Chryssolouris, 2013]. Therefore, as depicted in Figure 1, monitoring systems for the operator of an industrial plant have the intention to cover the current status of the assembly line including the involved car bodies, and occurring failures to ensure a fast reaction in case of interruptions [Zhang, 2010].

While the analysis of root causes of failures and quality deviations is usually built upon non-persistent, individual on-site expert knowledge, and hence troubleshooting relies on the individual knowledge of the staff on shift, advances of data-driven machine learning techniques have opened

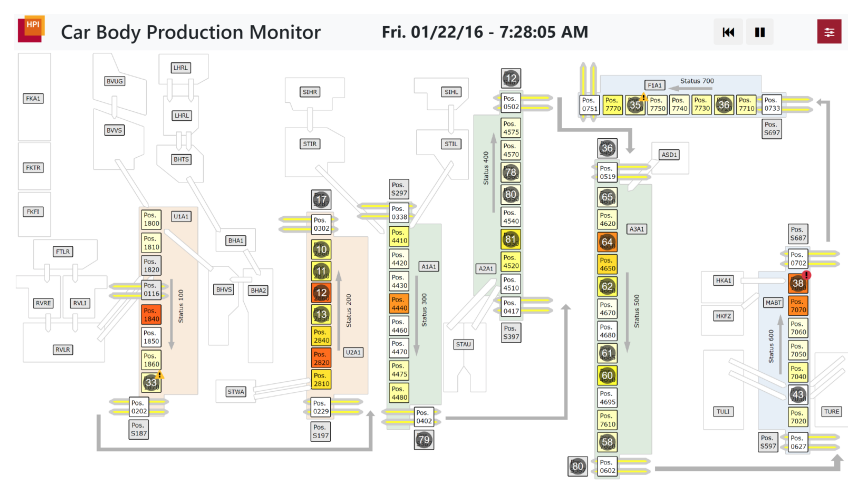

Figure 1: Monitoring View depicting the vehicles (dots) within the different production cells of the car body shop assembly line.

the possibilities to create monitoring applications that integrate failure diagnosis [Abellan-Nebot and Subirón, 2010; Liu et al., 2012]. Moreover, the emergence of methods for causal structure learning (CSL), e.g., see [Spirtes et al., 2000; Pearl, 2009], created the basis for attempts of a purely datadriven assessment of the causal structures from observational data of a manufacturing process [ $\mathrm{Li}$ and Shi, 2007; Marazopoulou et al., 2016]. While this previous work addresses challenges in domain-specific data preprocessing and examines the quality of the derived CGMs, it remains open to demonstrate how the knowledge about causal structures can be leveraged within the assembly line.

Consequently, a demonstration of the possibilities of incorporation of CGMs into the assembly lines for on-line monitoring, and failure diagnosis, also under the prism of making the troubleshooting more efficient, is of great importance.

\section{Causal Modeling of the Assembly Line}

This section covers the steps for learning the CGM from historic data and its parameterization for on-line prediction.

Production Process. An automotive body shop assembly line consists of multiple sections, e.g., responsible for attachments where the prefabricated individual parts are fitted to the car's body. Each section is separated into high-automated production cells in which robots weld, rivet or bend summing up to several hundreds of involved robots constantly streaming data, e.g., failures or quality measurements. 


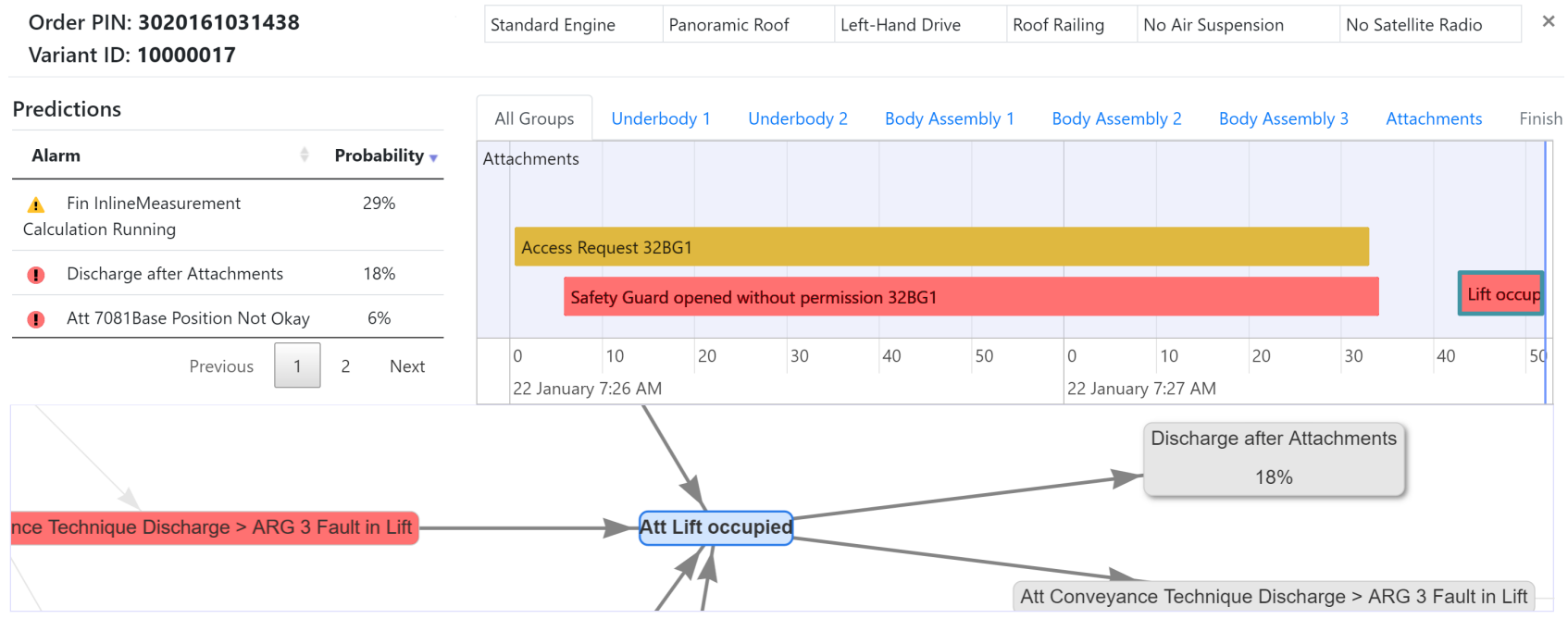

Figure 2: Detailed Failure View of a car body including failure history (top right), individual failure prediction (top left), and corresponding causal graphical model (bottom) given the current failure in the center with possible root causes to the left and subsequent failures to the right.

Data Preprocessing. The raw data comprises historic logged failure occurrences and quality measures, categorized by their severity, of a two year production time for one car body type. An iterative approach incorporating process metadata under consideration of domain knowledge yields to $70 \mathrm{k}$ sound discrete observations of $6.5 \mathrm{k}$ variables, e.g., according to [Li and Shi, 2007]. Each observation covers the failure and quality deviation history of a specific car body.

Causal Structure Learning. Given the historic observational data, the CGM is estimated through constraint-based CSL using the PC algorithm taking path constraints implied by domain knowledge into account, e.g., see [Spirtes $e t$ al., 2000; Borboudakis and Tsamardinos, 2012]. Hence, the CGM's edges between vertices represent causal relationships between failures or quality deviations whereof focused selections were evaluated by domain experts.

Failure Prediction. The parameterized CGM, e.g, see [Neapolitan, 2004], enables to calculate the conditional probability of failures and critical quality deviations given the current state of the car body's production process.

\section{Demonstration}

In this section, we showcase the application possibilities of our monitoring tool that incorporates the causal knowledge between failures and quality deviations, and demonstrate the opportunities concerning a more efficient troubleshooting.

As the occurrence of failures and quality deviations interrupting the highly automatized production process requires an instant human intervention of the technical staff it is essential to have an accurate view on the current state of the body shop assembly line. Hence, the Monitoring View, depicted in Figure 1, provides an entry point to the main sections of the assembly line where the currently produced car bodies are depicted within the production cells. While the darker highlighted production cells indicate an increased probability of critical interruptions, and hence should be called under the attention, the occurrence of warning signs at the car bodies directly refers to the occurrence of failures or quality deviations requesting an instant manual troubleshooting.

In this situation, where every minute of an unscheduled stopping results in loss of money the Detailed Failure View, see Figure 2, provides the CGM depicting all possible preceding root causes to the current failure - highlighted in blue - as well as the full failure history of the affected car body. The CGM incorporates possible root causes for the whole assembly line and additionally refers to actually occurred, yet oftentimes unnoticed, root causes - vertices highlighted in red. Thus, the technical staff receives treatment recommendation that outperforms the usually non-persistent, individual on-site expert knowledge. Moreover, given the currently observed failure the depiction of possible subsequent critical failures in the CGM, with their probability estimation, enables and guides the technical staff within a predictive troubleshooting. In order to provide a comprehensive examination of possible future failures and quality deviations, a list covers critical failure predictions based upon the knowledge about the causal structures and the car body's full history of occurred failures. This combination of early warning in both the Monitoring View and the Detailed Failure View, the direct identification of root causes through the incorporation of a CGM and the extension trough the prediction of subsequent failures builds the basis of a data-driven decision-support for on-line monitoring of automotive body shop assembly lines.

\section{Concluding Remarks}

In this work, we demonstrated the possibilities of an integration of causal structural knowledge into a modern automotive body shop assembly line. Thereby, we enriched a monitoring tool with a CGM representing the causal structures between failures and quality deviations of the production process to provide the operator of an assembly line with data-driven decision-support enabling fast and effective troubleshooting. 


\section{Acknowledgments}

The authors would like to thank the project partner and the project students for their work that built the basis of this tool.

\section{References}

[Abellan-Nebot and Subirón, 2010] Jose V. Abellan-Nebot and Fernando R. Subirón. A review of machining monitoring systems based on artificial intelligence process models. The International Journal of Advanced Manufacturing Technology, 47(1-4):237-257, 2010.

[Borboudakis and Tsamardinos, 2012] Giorgos Borboudakis and Ioannis Tsamardinos. Incorporating causal prior knowledge as path-constraints in bayesian networks and maximal ancestral graphs. In Proceedings of the 29th International Coference on International Conference on Machine Learning, ICML'12, page 427-434, Madison, WI, USA, 2012. Omnipress.

[Chryssolouris, 2013] George Chryssolouris. Manufacturing systems: theory and practice. Springer Science \& Business Media, 2013.

[Li and Shi, 2007] Jing Li and Jianjun Shi. Knowledge discovery from observational data for process control using causal bayesian networks. IIE transactions, 39(6):681690, 2007.

[Liang et al., 2004] Steven Y. Liang, Rogelio L. Hecker, and Robert G. Landers. Machining process monitoring and control: the state-of-the-art. J. Manuf. Sci. Eng., 126(2):297-310, 2004.

[Liu et al., 2012] Tenghong Liu, Rong Yuan, and Huating Chang. Research on the internet of things in the automotive industry. In 2012 International Conference on Management of e-Commerce and e-Government, pages 230233. IEEE, 2012.

[Marazopoulou et al., 2016] Katerina Marazopoulou, Rumi Ghosh, Prasanth Lade, and David Jensen. Causal discovery for manufacturing domains. CoRR, abs/1605.04056, 2016.

[Neapolitan, 2004] Richard E. Neapolitan. Learning bayesian networks, volume 38. Pearson Prentice Hall Upper Saddle River, NJ, 2004.

[Pearl, 2009] Judea Pearl. Causality: Models, Reasoning and Inference. Cambridge University Press, New York, NY, USA, 2nd edition, 2009.

[Spirtes et al., 2000] Peter Spirtes, Clark N. Glymour, and Richard Scheines. Causation, Prediction, and Search. MIT press, 2000.

[Zhang, 2010] Peng Zhang. Chapter 2 - industrial control engineering. In Peng Zhang, editor, Advanced Industrial Control Technology, pages 41 - 70. William Andrew Publishing, Oxford, 2010. 